\title{
Optimization of Second-Order Grey-Level Texture in High-Resolution Imagery for Statistical Estimation of Above-Ground Biomass
}

\author{
Y. O. Ouma ${ }^{1 *}$ and R. Tateishi ${ }^{2}$ \\ ${ }^{1}$ Centre for Environmental Remote Sensing, Graduate School of Science and Technology, Chiba University, \\ 1-33 Yayoi, Inage, Chiba 263-8522, Japan \\ ${ }^{2}$ Centre for Environmental Remote Sensing, Chiba University, 1-33 Yayoi, Inage, Chiba 263-8522, Japan
}

\begin{abstract}
In this paper, part of the Mt. Kenya forest with mixed vegetation biophysical characteristics was selected for grey-level co-occurrence matrix (GLCM) optimization and comparison based on semivariogram modeling from high spatial resolution QuickBird imagery. The results were applied to demonstrate the role of GLCM-textures in the estimation of Above-Ground Biomass (AGB) for: the dominant afromontane (camphor) trees, tea, young and old planted pine trees from QuickBird imagery. The texture optimization results were compared and combined with spectral (near-infrared) information for AGB estimation. To quantify the significance of GLCM-textures in AGB estimation, regressions between the field-AGB estimates and estimates from the NIR band and the tested GLCM-textures as independent variables, and their integration as dependent variables were compared. As independent variables, NIR and variance-texture bands gave the best results for the dominant camphor trees, with accuracies of 72 and $67.34 \%$ respectively. Variance and mean textures gave the best results upon combination with NIR, showing an improvement of 4.33 and $4.82 \%$ respectively over the NIR estimates. For tea, the combination of NIR with homogeneity, entropy and second moment textures gave the best and equal results $\left(R^{2}=0.684\right)$. For the young pine trees, correlation texture gave the overall best results $\left(R^{2}=0.741\right)$, and for the older pine trees, contrast texture gave the best results $\left(R^{2}=0.753\right)$ as independent variables. We conclude that the role of texture type and optimal window in AGB estimation depends on the: size (height), age, species, inherent spatial structure (natural or planted) and crown size of the vegetation species.
\end{abstract}

Keywords: Above-Ground Biomass (AGB), GLCM-texture optimization, QuickBird, semivariance, vegetation-trees

\section{Introduction}

Biomass determines potential carbon emission that could be released to the atmosphere due to deforestation or conversion to non-forest land use. Forest biomass or the dry mass of live plant material is a measure used in studies of forest ecosystem processes and in models that calculate or forecast carbon budgets. Estimation of Above-Ground Biomass (AGB) is becoming significant because the change of biomass regionally is associated with important components of climate change. AGB estimation for tropical forests is necessary for studying productivity, carbon cycles, nutrient allocation, and fuel accumulation in terrestrial ecosystems (Brown et al., 1999; Ryu et al., 2004). Accurately quantifying the role of tropical forests in the global carbon cycle is one of the key requirements to improve our understanding of current patterns of terrestrial carbon exchange. In particular, the estimates of forest biomass used in models of carbon flux in tropical regions, are an important source of uncertainty. Therefore, accurate biomass estimation is necessary for better understanding of deforestation impacts on global warming and environmental degradation. Computer-processed remotely sensed data are possibly the best data for economical AGB estimation in the tropical re-

\footnotetext{
* Corresponding author: yashon@graduate.chiba-u.jp
}

gions over large areas.

Many studies have demonstrated that the indices such as spectral vegetation index (SVI), simple ratio (SR), normalized difference vegetation index (NDVI), and corrected normalized difference vegetation index (NDVIc) obtained from satellite data are useful predictors of leaf area index (LAI), biomass, and productivity in grasslands and forests (Jakubauskas, 1996; Paruelo and Lauenroth, 1998; Steininger, 2000; Chen et al., 2002). Stand level biomass is frequently calculated from linear and nonlinear regression models established by species with field measurements. Although the estimates of AGB vary with species composition, tree height, basal area, and stand structure, bole diameter at breast height (DBH) is the most commonly used and widely available variable for calculating AGB (Crow and Schlaegel, 1988).

Previous studies have shown varying degrees of success in estimating forest biomass and primary production from remote sensing data in temperate and tropical forests worldwide (Jakubauskas, 1996; Lee and Nakane, 1997; Brown et al., 1999; Steininger, 2000; Sannier et al., 2002; Chen et al., 2002). Recent studies suggest that such relationships vary temporally and spatially; however, the biomass estimates at the landscape level are necessary for understanding processes of the target landscapes and provide baseline data for future studies (Foody et al., 2003). 
(a)



(b)

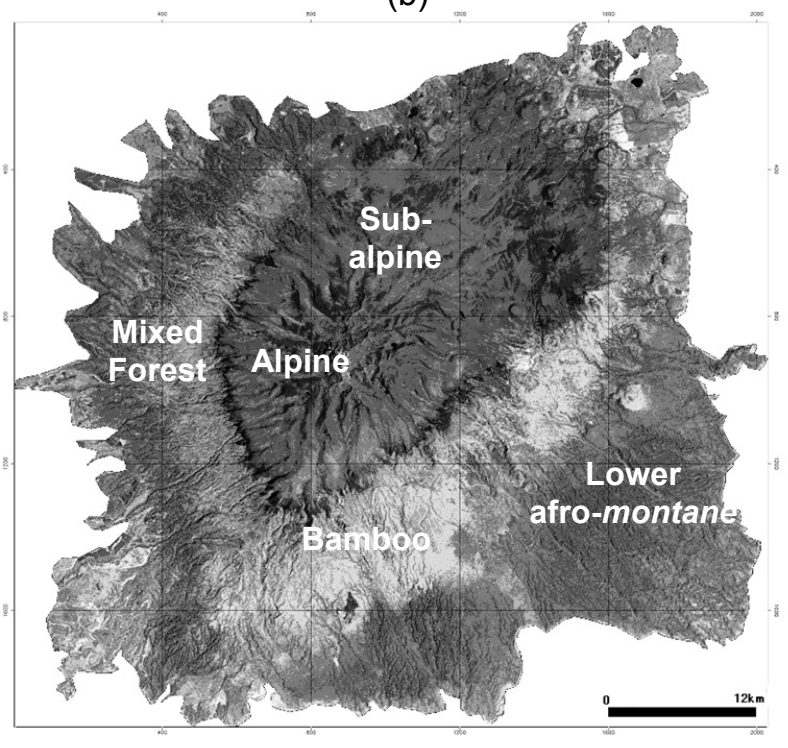

Figure 1. (a) Location of the study site; (b) 543 false color composite of Landsat ETM+ of the study area.

Image texture (Olthof and King, 1997), has been suggested in improving the forest biophysical parameters estimation, however mostly at medium resolutions of Landsat or SPOT data. With the advent of the very high spatial resolution sensors like IKONOS and QuickBird, the existing techniques for AGB estimation in the medium-resolution sensors may not apply, since at these high resolutions, we are considering individual trees (forest biotic parameters and species composition) and not patches (Bailey et al., 2001) as in Landsat or SPOT, for example.

The launch of the QuickBird satellite, with $0.61 \mathrm{~m}$ panchromatic and $2.44 \mathrm{~m}$ multispectral 11 bit data, ushers in a new era in civilian remote sensing. The QuickBird characteristics provide an opportunity for capturing quantitative parameters of floristic structural and species composition changes across the small topographic gradients at very fine spatial resolution (0.61 to $2.44 \mathrm{~m}$ ) enabling capture of spectra from individual units such as a tree crown. High resolution images have demonstrated the possibility of extracting the location of individual tree crowns from $1 \mathrm{~m}$ panchromatic data to produce image maps representing stem densities and crown cover densities (Read et al., 2003).

Although various methods for biomass estimation have been tested, as already mentioned, rarely has research at very high spatial resolutions been successfully conducted in moist tropical rainforest regions due in part to the resulting complex tropical forest stand structure and abundant tree species. The previous research used mainly spectral signatures to establish biomass estimation models (Franklin and Hiernaux, 1991; Nelson et al., 2000; Steiniger, 2000), but ignored spatial infor- mation largely due to lack of understanding about the relationships between biomass and spatial characteristics (texture).

Image spatial information (texture) is an important factor in improving biomass estimation accuracy even though many uncertainties exist at high spatial resolutions. For example, what types of texture are appropriate to extract biomass information? What size of moving window for selected texture measures can most effectively extract biomass information? Which models can be used to estimate biomass using remote sensing technology? Is there a specific or general role played by texture in the estimation of AGB at high spatial resolutions. Because of the complex stand structures and abundant vegetation species at high-spatial resolution remote sensing data, the role of texture in Above-Ground Biomass (AGB) estimation in tropical forests ought to be understood.

In order to explore biomass-texture relationship in high spatial remote sensing data, QuickBird image of part of Mt. Kenya forest was studied. Grey-level co-occurrence (GLCM) textures were computed and optimized using semivariogram geostatistics for optimal texture window determination. The optimization results were used to demonstrate the significance of texture and spectral information, as independent variables and as combined variables, for AGB estimation for afromontane (camphor) tree. With this approach, the suitable texture type(s) for this case study was also revealed. The overall objective of this study was to compare field observed AGB and that derived form remote sensing data in order to establish a background for understanding: (1) the relationships between texture and AGB in high resolution imagery, and (2) the influence of combining the high resolution image spatial variables 
with its spectral variables in AGB estimation.

\section{Study Area}

The study area is located on Mt. Kenya in Kenya (Figure 1). Mt. Kenya, located in central Kenya at approximately $0^{\circ}$ $09^{\prime} \mathrm{S}, 37^{\circ} 18^{\prime} \mathrm{E}$, is the second largest mountain $(5,199 \mathrm{~m}$ above mean sea level, AMSL) in Africa after. The approximated boundary of Mt. Kenya encloses a total area of about 3,132.56 $\mathrm{km}^{2}$, and includes the top sub-alpine and alpine belts of the mountain and other forest cover as shown in Figure 1b. The heterogeneity of the landscape within this site is extreme both between and within land use and land cover types.

Due to the wide range of altitude that spans the indigenous forest (from $1,200 \mathrm{~m}$ to $3,400 \mathrm{~m}$ ) and the major climatic differences between the slopes, the forest vegetation of Mt. Kenya is characterized by a high diversity of forest types and various vegetation zones can be distinguished on the Mount Kenya (Figure 1b). For example, the natural vegetation ranges from sparse tundra vegetation, bamboo and afromontane rainforest, to sparse grasslands in the lower elevation, dryland area, with altitudes ranging $600 \mathrm{~m}$ AMSL.

Figure 2a shows part of the QuickBird bands 321 false color composite image that was used in this study. It covers the southeastern portion of the ETM+ image shown in Figure $1 \mathrm{~b}$. The predominant land cover is the afromontane (camphor trees) broadleaf forest. Non-forest land cover like tea and coffee plantations have over the years replaced some of these indigenous species or primary forest cover. In efforts to conserve the forests, needle-leaved forests have been planted mostly around the plantations. Logging of camphor in this locale has given way to open grasslands and sometimes bare soil as seen in Figure 2. In Figure 2b, four representative landcover types within the study site are shown: (i) deciduous afro-montane forest (camphor trees), (ii) planted coniferous trees (young and old) on the lower part of the image and tea plantations on the upper part of the image, and (iii) logged area with grass and other understory. Figure $2 b$ manifests the intensity of afforestation and deforestation within the lower parts of the most fertile south eastern (SE) sections of mountain.

In terms of area and stocking of species, the montane forest zone forms the most important zone. It contains the highest mean total volume per ha with standing volume estimated at $253 \mathrm{~m}^{3} / \mathrm{ha}$ and an average timber volume of $61 \mathrm{~m}^{3} / \mathrm{ha}$, in the eastern side of the forest. In the more accessible areas the natural vegetation is sometimes converted into plantation forests. The remaining undisturbed primary forests montane forest trees are mainly confined to the deep inaccessible areas. Within the western rainforest region, the standing volume is estimated at $230 \mathrm{~m}^{3} / \mathrm{ha}$ and the mean timber volume at 78 $\mathrm{m}^{3} /$ ha.

Our research focuses on the most significant and primary species on Mt. Kenya, which is the afromontane forest dominated by camphor species. The afromontane (camphor) hardwood tree is a multiple-use tree species with high economic and medicinal values, primarily for its bark (traditional medicines) and hard timber, and is the main contributor to the ecosystem stability not only within the Mt. Kenya forest, but also in the entire region around the mountain.

\section{Materials and Methods}

\subsection{Data Collection and Analysis}

The satellite data used in this study were the pan-sharpened multispectral QuickBird channels: blue: 450 to $520 \mathrm{~nm}$; green: 520 to $600 \mathrm{~nm}$; red: 630 to $690 \mathrm{~nm}$; near-IR: 760 to $900 \mathrm{~nm}$. These channels were resampled from $0.61 \mathrm{~m}$ using nearest-neighbor convolution to $1 \mathrm{~m}$ spatial resolution. A nested sampling strategy, organized by region, site and subplot, was employed to collect field data, which was primarily to select sample land cover/trees for the proposed texture and biomass estimation experiments. In this report, the focus is on the experimental plot that consists of the afromontane (camphor) trees. Landsat ETM+ and differential global positioning system (DGPS) devices were used during the fieldwork. Plots were designed to inventory afromontane trees, and subplots were used to inventory the different forestland cover as shown in Figure 2. These land cover representatives are referred to here as "training samples". The size of these samples was selected at $23 \mathrm{~m} \times 23 \mathrm{~m}$ to represent an entire camphor tree, with minimum noise. The same size was applied to the other samples, for experimental purposes. At the high spatial of QuickBird, the outline or crowns for the trees and the nontrees are visible clearly identifiable, as in Figures $2 a$ and $2 b$. This mapping capability allowed for more precise and easy measurement of 2D-physical parameters like the crown size of these trees from the imagery. In the experimental plot, the diameter at the breast height $(D B H)$ were identified and measured and also the total tree height $(H)$.

The "training samples" represented in Figure 2 were used in assessing the behavioral characteristics of the co-occurance texture, with respect to window size, texture type and utility of texture in AGB estimation. The natural and dominant afromontane (camphor) trees were compared against planted trees of pine species (both young and old) as well as tea plantations, logged (understory), bare soil, grass and clouds with respect to texture characteristics. Cloud was used here only to analyze the hypothetical characteristics of " $100 \%$ pure" or homogenous surface spatial signature. The spectral signatures of the "training samples" were compared between: blue, green, red, NIR bands and NDVI $(=(N I R-r e d) /(N I+r e d))$.

From the plot of the spectral patterns of all training samples, it was observed that all the patterns or signatures appeared to be similar except for clouds and bare soil, which of course have no green-matter (chlorophyll). The spectral narrowness and confusion in the visible bands was evident, depicting the fact that the visible bands are by themselves obviously not suitable for biophysical parameters extraction. It is noted that even in the NIR and NDVI where vegetation is supposed to be most sensitive; there is strong evidence of high spectral confusion. NIR is the only band that appeared to pre- 


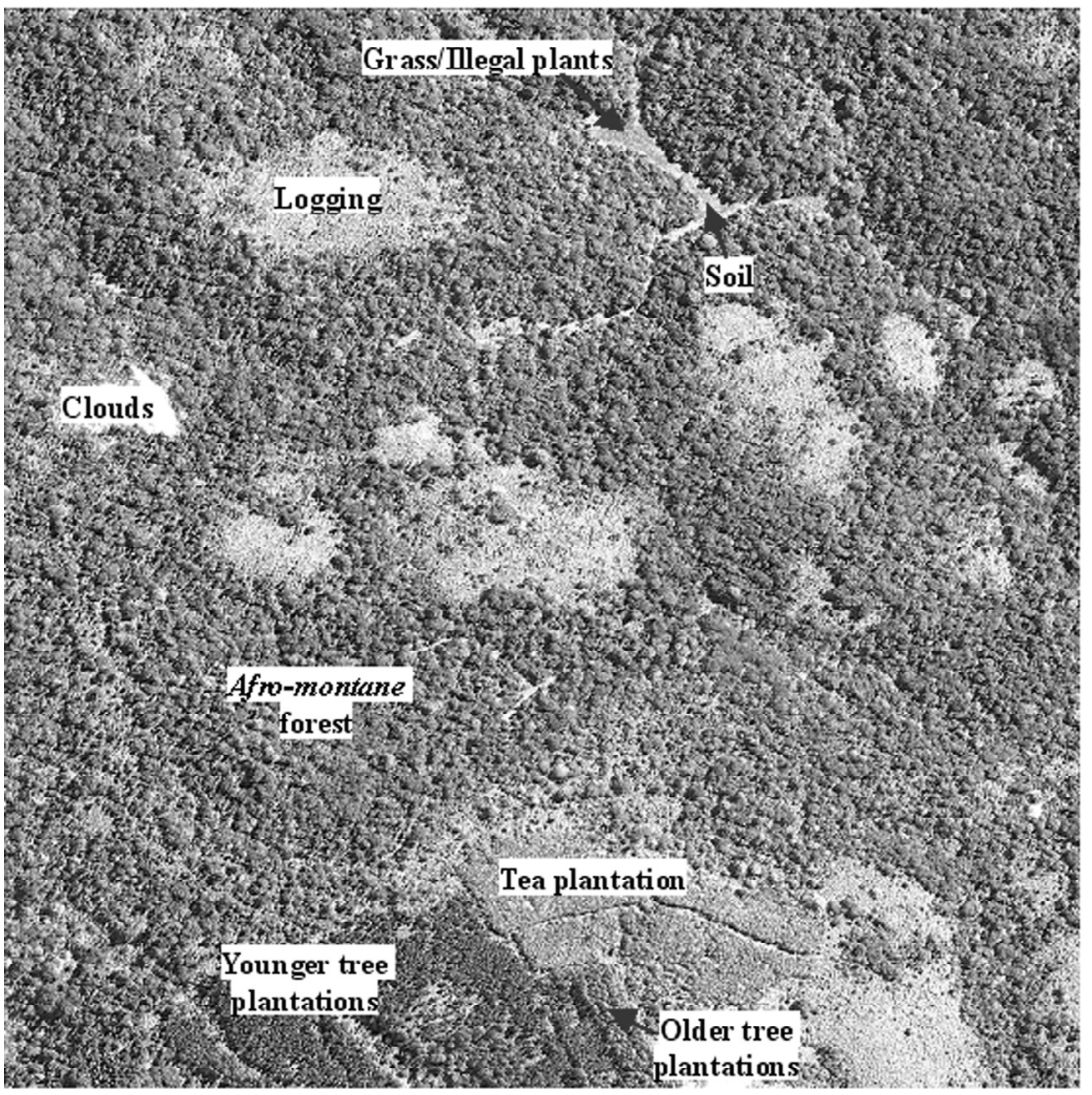

(a)

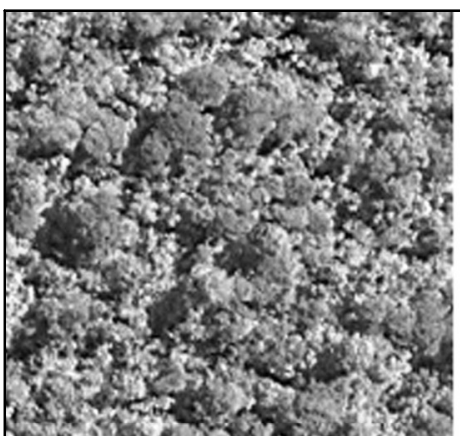

(i)

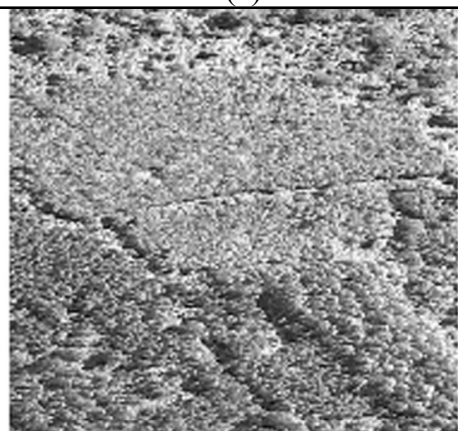

(ii)

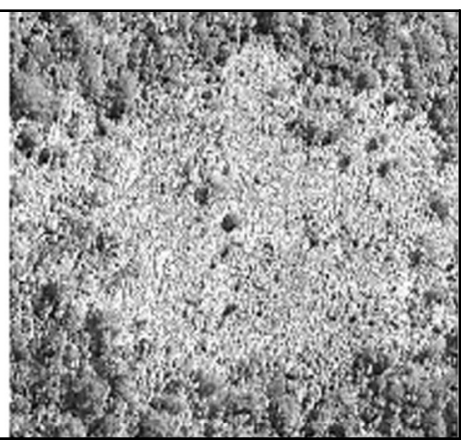

(iii)

(b)

Figure 2. (a) False color composite of QuickBird bands 321 showing the selected test site; (b) Sample vegetation land cover within the forest test-site.

sent different spectral behavior for all the 8-training samples. The NDVI is not used for further analysis as would prompt the questions of comparing it with other similar vegetation indices. These observations imply that the spectral signatures alone may not be wholly relied upon for accurate separation of these ground cover and even AGB estimation. Therefore this study suggested the combination of spectral patterns with spatial characteristics (texture) in AGB estimation, as argued in the introduction.
To determine which of the QuickBird bands available for research is suitable for spatial information extraction, we plotted the regressions between the NIR band versus the blue, green and red bands. The following regressions with the NIR band were obtained: (a) 0.71 (blue), (b) 0.85 (green) and (c) 0.79 (red). All the visible bands show high correlation $\left(R^{2}>\right.$ 0.70) with the NIR thus band supporting the observations from spectral pattern plots, that NIR is the best band for texture extraction in this particular case. 
From the above observations, it was concluded that the texture or spatial pattern structure of the vegetation classes in this scene are resident in the NIR band. Arguing that the texture depends on the spatial resolution, the higher the spatial resolution, the better is the texture information revealed in an image. Also for reliable texture, one must select the suitable spectral band(s). Panchromatic band has been widely used for texture analysis in higher spatial resolution imagery. However in the vegetation analysis the wavelength of the panchromatic band is considered to be too wide and contains extra information that can be viewed as noise for vegetation texture extraction. Since the current study is concerned with texture window and type optimization, we do not detail further on the selection of suitable texture band(s).

\subsection{GLCM Texture Extraction}

First-order statistics measures the likelihood of observing a grey value at a randomly chosen location in the image. Firstorder statistics can be computed from the histogram of pixel intensities in the image. These depend only on the individual pixel values and not on the interaction or co-occurrence of neighboring pixel values. The average intensity in an image is an example of the first-order statistic. Second-order statistics on the other hand are defined as the likelihood of observing a pair of grey values occurring at the endpoints of a dipole (or needle) of random length placed in the image at a random location and orientation. These are properties of pairs of pixel values. Co-occurance measures do not assess the texture of the image directly; rather, they assess patterns that arise in the matrix formed by the juxtaposition of particular tonal values or classes. In this study, the co-occurance texture was selected due to its inter-pixel spatial information extraction properties. The application of a set of textural measurements to remotely sensed data began with the second-order textural measures based on Haralick's grey level co-occurance matrix (GLCM) (Haralick et al., 1973). Distance and angular spatial relationships among grey levels are summarized in a GLCM, as it is a measure of the probability of occurance of two grey levels separated by a given distance in a given direction.

Traditionally, texture methods have been evaluated over windows of a single size, the latter being commonly defined on experimental basis. The role played by both the shape and size of evaluation windows was studied in (GarcíaSevilla and Petrou, 2000), showing that texture characterization is much more influenced by window size than by its shape. Although many studies regarding the performance of the different families of the texture feature extraction methods have been carried out in the past (e.g. Chang et al., 1999; Randen and Husoy, 1999), only a few have dealt with the issue of determining optimal window sizes. The majority of these works are actually outside the scope of land cover mapping. In the scope of pixel-based AGB estimation from high-resolution imagery, a technique for determining the optimal window size that leads to the best possible estimator among different GLCM texture models of interest is necessary. Determining optimal GLCM windows via trial-and-error methods are time consuming and may not always be reliable.

An optimal window size for calculating GLCM texture measures is a compromise between providing enough spatial information to characterize the land cover and limiting overlapping textures between different land covers. No rules have been recommended for the texture measures selection. The most appropriate combination of the texture features depends strongly on the surface properties of the land cover types of interest. Since unique texture patterns are hypothesized to discriminate different land cover types, a proper window size that matches the patch size can extract the textural pattern of this particular landscape. Large window size can capture the spatial patterns of each land cover type better, but may contain more than one land category, which could introduce systematic errors. The window should then be small enough to keep the variance low and to maximize the potential for class separability.

\subsubsection{Texture measures from GLCM}

Arguably, due to the different landscape feature interactions at different image spatial resolutions, different texture windows and types will obviously result for the same scene. This step of the study is thus trivial in the analysis of any imagery. To determine the spatial information present in a digital image, a co-occurance matrix is computed on a pixel neighborhood delimited by a moving window of a given size. Each element $P(i, j, d, \theta)$ of the co-occurance matrix represents the relative frequency with which two neighboring pixels (separated by a distance $d$ and having an angular relationship $\theta$ ) occur on the image, one with grey level $i$ and the other with grey level $j$. Subsequently, statistics are computed from the grey level co-occurance matrix and they describe the spatial information according to the relative position of the matrix elements. The 8-texture measures analyzed in the work are summarized in Table 1: mean, variance, homogeneity, contrast, dissimilarity, entropy, angular second moment and correlation. A more complete theoretical description of the most commonly used co-occurance measures can be found in (Haralick et al., 1973; Soares et al., 1997).

\subsubsection{Evaluation of texture methods over multisized windows}

Let $\left\{\tau_{1}, \tau_{2}, \ldots, \tau_{T}\right\}$ be a set of $T$ texture models of interest. Each model $\tau_{T}$ is described by a sample image $I_{k}$ that contains a pattern of that texture. Let $I$ be a two-dimensional test image of $R \times C$ pixels that contains several regions of uniform texture. The usual way of classifying each pixel $I(x, y)$ consists of computing a texture feature $f$ obtained by applying a texture feature extraction method $\mu$ to the pixels contained in a neighborhood of $I(x, y): f=\mu(x, y)$. That neighborhood is usually a square window centered at $I(x, y)$ whose size is experimentally set for each method. The computed feature is then fed into a pattern classifier in order to determine the texture model corresponding to $I(x, y)$.

In Table 1: 
Table 1. The GLCM Texture Tested in This Study

\begin{tabular}{ll}
\hline Co-occurance texture and formula & Description \\
\hline Mean & Mean is the average grey level in the local window. \\
$M E A N_{\Delta x \Delta y}=\sum_{i} i^{*} p(i)$ &
\end{tabular}

Variance

$V A R_{\Delta x \Delta y}=\sum_{i} \sum_{j}\left(i-\mu_{i}\right)^{2} * P(i, j)$

Entropy

$E N T H_{\Delta x \Delta y}=-\sum_{i} \sum_{j} P(i, j)_{\Delta x \Delta y} \log P(i, j)_{\Delta x \Delta y}$

Angular Second Moment (ASM)

$A S M_{\Delta x \Delta y}=\sum_{i} \sum_{j} P(i, j)^{2} \Delta x \Delta y$

Homogeneity

$H O M_{\Delta x \Delta y}=\sum_{i} \sum_{j} \frac{P(i, j)_{\Delta x \Delta y}}{1+(i-j)^{2}}$

Contrast

$C O N T_{\Delta x \Delta y}=\sum_{i} \sum_{j}(i-j)_{\Delta x \Delta y}^{2} P(i, j)_{\Delta x \Delta y}$

Dissimilarity

$$
D I S_{\Delta x \Delta y}=\sum_{i} \sum_{j} P(i, j)_{\Delta x \Delta y}|i-j|
$$

Correlation

$\operatorname{COR}_{\Delta x, \Delta y}=\frac{\sum_{i} \sum_{j}\left(i-\mu_{i}\right)\left(j-\mu_{j}\right) P(i, j)_{\Delta x \Delta y}}{\sigma_{i} \sigma_{j}}$
Grey level variance in the local window. High when there is a

large grey level standard deviation in the local region.

Entropy is a measure of the degree of disorder in an image. Entropy is larger when the image is texturally non-uniform or heterogeneous and approaches its maximum when all GLCM entries have similar contents, indicating an image with completely random pixel values. High entropy when GLCM have relatively equal values and low when the elements are close to either 0 or 1 .

Also called energy, angular second moment and uniformity is a measure of textural uniformity or pixels pairs repetitions. When the pixels of the image window under consideration have similar grey levels, energy reaches its maximum (equal or close to 1). Therefore, constant or periodic distribution of grey levels over the window will produce high values for energy. It is high when GLCM has few entries of large magnitude, when all entries are almost equal. This is the measure of the local homogeneity. Entropy and ASM are inversely correlated.

Also called inverse difference moment, homogeneity is a measure of lack of variability or the amount of local similarity in the scene. High homogeneity values suggest small grey tone differences in pair elements. In this case, the associated GLCM will present elements around the main diagonal. Homogeneity is high when GLCM concentrates along the diagonal. This occurs when the image is locally homogenous in the scale of the length of spatial

Contrast is a measure of the degree of spread of the grey levels or the average grey level difference between neighboring pixels. The contrast values will be higher for regions exhibiting large local variations. The GLCM associated with these regions will display more elements distant from the main diagonal, than regions with low contrast. Contrast is high when the local regions have a high contrast in the scale of spatial. Local statistics contrast and GLCM contrast are strongly correlated. Contrast and homogeneity are inversely correlated.

Similar to contrast. High when the local region has a high contrast.

Correlation is a measure of grey level linear dependencies in the image. High correlation values denote a linear relationship between the grey levels of pixel pairs. A completely homogeneous area is a limiting case of linear-dependency, for which correlation reaches its maximum (equal to 1).

Correlation is uncorrelated to entropy and energy, i.e., to pixel pair repetitions. 
$p_{x}(i)=\sum_{j} p(i, j)$ and $p(i, j)=\frac{P(i, j)}{\sum_{j} \sum_{j} P(i, j)}$

Each element $P(i, j)_{\Delta x \Delta y}$ represents the relative frequency with which two neighboring pixels separated by a distance of $\Delta x$ columns and $\Delta y$ lines occur (Soares et al., 1997).

Instead of using a single window, we propose the evaluation of the given texture method $\mu$ over $N$ square windows, $\left\{w_{1}, w_{2}, \ldots, w_{N}\right\}$, with each window having a different size. Every window $w_{j}$ is considered to contain $s_{j} \times s_{j}$ pixels, with $s_{j}$ $=2^{j}+1$. Hence, every texture method $\mu$ generates a feature vector with $N$ texture features for every pixel to which the method is applied. We consider that whenever an evaluation window is not totally contained in the given image, the texture method cannot be evaluated, since it would generate a value based on a fraction of the texture pattern. This means that the strip of pixels that belong to the boundary of $I$ will not be analyzed, as no window centered at them will entirely fit into the image. Let $W, W \leq N$, be the number of windows that do entirely fit into the image for a specific pixel $I(x, y)$. In this case, $\mu$ generates a vector $F$ of $W$ features: $F=\left(f_{1}, f_{2}, \ldots, f_{w}\right)$. To evaluate the window sizes, the selected homogenous "training samples" shown in Figure 2 are used. Eight GLCM texture measures described in Table 1 are computed for 20-window sizes ranging from $3 \times 3$ to $41 \times 41$. We chose these window ranges as $3 \times 3$ is the minimum possible texture mask and 41 $\times 41$ approximately corresponds to the largest tree crown size within the study site.

Assuming the "training samples" are homogenous and that they accurately represent the ground cover, then theoretically it implies that the best GLCM texture should have the least variance over the given training sample image. Thus the variance for each of the tested GLCM texture type was computed over the test windows and compared for each training sample. This optimal texture measure is then used to determine the optimal window size for each of the "training sample" using variogram geostatistical method. The derived optimal window is assumed to be the same for the rest of the GLCM texture measures.

\subsection{Multisized Texture Window Optimization Using Variogram Modeling}

The variogram is derived by calculating half the average squared difference (semivariance) between pairs of pixels separated by a distance $h$ (the lag distance). Variograms are characterized by variation in the sill, range and nugget. The sill is the background level of variance among samples. The range is the inter-core distance at which the fitted curve reaches the sill, indicating the distance over which spatial autocorrelation (i.e., patchiness in occurance of species) can be detected. The nugget variance ( $y$-intercept of the fitted line) is the variance unexplained by the fitted curve. Geostatistics is currently a well-understood and frequently applied image processing technique: it has been shown that range is directly related to the texture and/or objects size, while sill is proportional to object (class) variance.

The spatial scales at which "training samples" can optimally be mapped were determined using standardized variograms; with the geostatistical program Variowin 2.2 (Pannatier, 1993 - 1996). The Spherical, Gaussian and Exponential models were fitted to all the samples and the model with the best fit selected. The average change of a property is illustrated by a changing lag and the classical equation of the semivariogram $\gamma(h)$ can be expressed as follows:

$\gamma(h)=\frac{1}{2 N(h)} \sum_{i=1}^{N(h)}\left[Z\left(x_{i}\right)-Z\left(x_{i}+h\right)\right]^{2}$

where $Z$ is DN value, and $x$ is the DN location, and $N(h)$ is the number of pairs of locations separated by the lag distance $h . h$ is a vector in both distance and direction. In our case we used the omnidirectional condition to avoid directional biasness. The properties of typical variogram and main descriptors of the semivariogram used are illustrated in Figure 3.

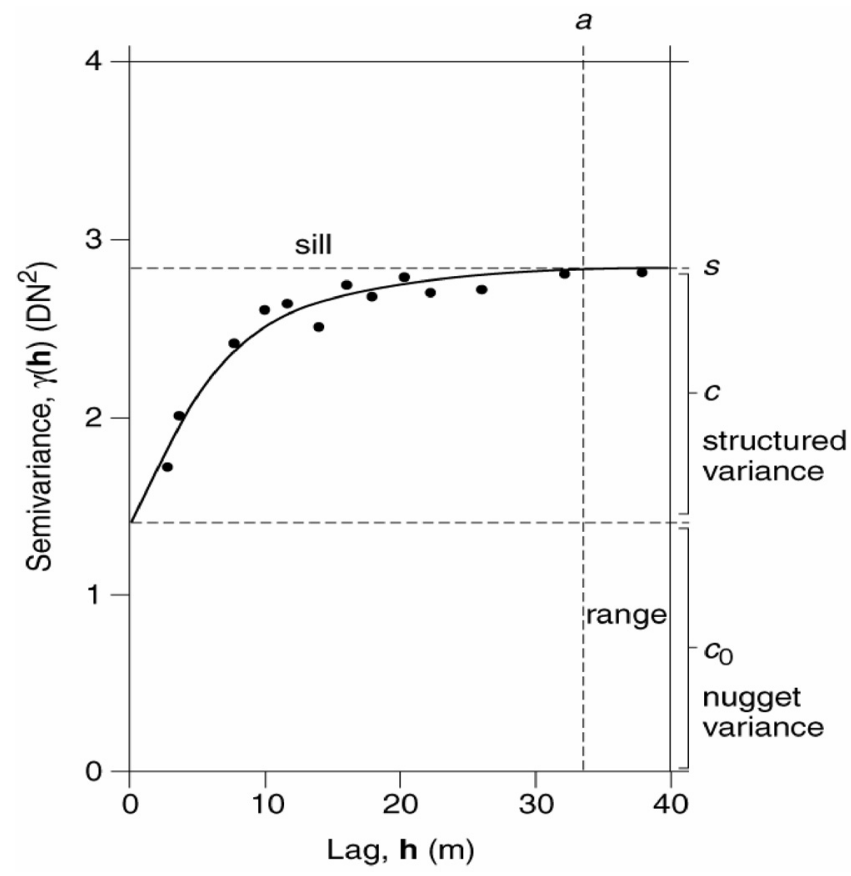

Figure 3. A variogram and its main descriptors (Curran and Atkinson, 1998).

The following spherical model was fitted to the semivariograms:

$\begin{cases}\gamma(h)=C_{0}+C\left(\frac{3}{2} \frac{h}{a}-\frac{1}{2} \frac{h^{3}}{a^{3}}\right) & \text { as } h \leq a \\ \gamma(h)=C_{0}+C & \text { as } h \geq a\end{cases}$ 
where $C_{0}$ is the nugget, $\left(C_{0}+C\right)$ is the sill, and $a$ is the correlation length. The resulting variogram is used for the fit of a model with known mathematical properties. The quality of the semivariogram fit to the data was indicated by using regression $\left(R^{2}\right)$ and an $F$-test calculated as:

$F=\frac{R^{2}}{1-R^{2}} \times \frac{N-k}{k-1}$

where $N$ is the number of samples and $k$ is the number of variables in the regression model. The semivariograms of the eight land cover classes were fitted and the qualities examined. From the semivariance modeling results, the optimal windows were determined for each training sample class. In this study, as opposed to most texture and spectral information integration for land cover classification studies; we utilize the optimized texture in estimating the AGB for afromontane trees.

\subsection{Integration of AGB and Remotely Sensed Data}

Remote sensing data are comprehensive responses of the vegetation stand structure, vegetation density and vegetation species composition. Different forest stand structures have different reflectance and texture patterns in various wavelengths, and the relationship between AGB and remote sensing data are different. The Pearson's correlation may be used to analyze such relationships. It measures the strength of linear relationships between two variables. If the coefficient is close to 1 , it implies there is a strong relationship between the variables. In this study, one variable is AGB; the other one is remotely sensed data-NIR and texture measure. Using biomass as dependent variable and remotely sensed data as the independent variable, regression computations are used to establish the relationships between the AGB and the remotely sensed data independently and upon integration (texture + NIR). The critical step here is to find the appropriate independent variables, so that combination of multiple independent variables could provide the best results.

The coefficient of regression $\left(R^{2}\right)$ is an indicator that can be used to determine whether or not the estimation is good enough, since $\left(R^{2}\right)$ measures the percent of variation explained by the regression model. Stepwise regression was used to find the best independent variable and also the best combined variables between the optimal texture window size and the NIR band. A single tree of the dominant afromontane (camphor) forest cover was used in the demonstration of the results of this phase of the study. This choice was made because of the relatively large area size (approximately $530 \mathrm{~m}^{2}$ ) covered by the tree. This area was considered as appropriate experimental size and also because the selected tree was a good sample representative of the afromontane (camphor) forest-cover trees. Also due to conservation and accessibility limitations, it was advised against felling of these valuable trees.

The allometric model $\left(\mathrm{Y}=\mathrm{a} \mathrm{X}^{\wedge} \sup \mathrm{b}^{\wedge}\right)$, in which $\mathrm{X}$ can be measured such as diameter at breast height (DBH), or DBH ${ }^{\wedge}$ sup $2^{\wedge} \mathrm{x}$ total tree height, and $\mathrm{Y}$ is tree component biomass such as foliage, branch, stemwood or stembark, has been widely used in biomass studies. Both the linear form $[\operatorname{In}(\mathrm{Y})=\mathrm{A}$ $+\mathrm{BIn}(\mathrm{X})]$ and nonlinear form $\left(\mathrm{Y}=\mathrm{aX}^{\wedge} \sup \mathrm{b}^{\wedge}\right)$ of the allometric model can be used to develop biomass equations for different tree species. The precision of such allometric equations may be good for stem biomass but is often unsatisfactory for canopy biomass components, like in this case study. Other variables, such as stand age, stand density, crown geometry, and site index, which influence biomass production and allocation, may need to be added to the model to improve the accuracy for these components. The process of biomass estimation is well established and thus not explained in details in this paper.

Preparation of biomass regression equations for forest trees is expensive and time-consuming. Many biomass equations have been published over the past two decades, and it is therefore natural that practicing foresters and ecologists on limited budgets will want to use them to estimate individual stand biomass or regional biomass from a large-scale survey. However, biomass estimates for a particular stand obtained using published equations can vary greatly according to which equation is used. This results from differences in stand density, stand development history, and tree growth rates (due to differences in climates and soil, for example) between the stands on which the equations are based and the stands to which they are being applied. Substantial errors in biomass estimation could be produced when biomass regression equations established at a given and in one area were applied to another.

In this study, the importance of accurate tree biomass is taken into account. For this reason, we did not rely on the existing biomass estimation allometric equations in this area, but rather on actual physical tree biomass estimation. Within a given plot, different sized afromontane trees were identified and a single tree used to calculate the biomass. The physical parameters of the trees were measured and used to correlate with the actual biomass content. The objective of identifying different-sized camphor trees was to be able to rigorously verify the AGB estimates from the single chosen tree through prediction or interpolation. For example, the tree height was predictable from the DBH. Crown diameters could also be related to height and DBH of the camphor tree samples. The results of these correlations are not relevant to the current research objectives hence detailed thereof are omitted.

Briefly, for biomass measurements of the sample trees, after felling, the live crown of afromontane sample tree was proportioned into three sections of equal length along the bole. All branches with leaves and twigs attached were removed from the main stem and weighed fresh. A 10\% subsample of the crowns of trees with $\mathrm{DBH}>55 \mathrm{~cm}$ was taken; for trees with DBH less than $55 \mathrm{~cm}, 100 \%$ of the crown was taken. All leaves were separated from the sampled branches. The fresh weight of all tree components was determined in the field. Subsamples of leaves, live and dead branches, stemwood, and bark were oven-dried at $70{ }^{\circ} \mathrm{C}$ to determine field moisture content, which was used to convert fresh weight to dry weight. Stem discs cut from the bottom of each stem section were measured to obtain the moisture content, specific gravity and 
bark/wood ratio. Stem volume of each stem section was measured using Smalian formula. The dry weight of stemwood and stembark were calculated on the basis of the above measurements. Cumulative biomass of the test trees was then obtained and compared for the other sized trees. Through interpolation, the actual biomass for selected tree was then estimated. As already stated, only one test tree was investigated in detail to demonstrate biomass-texture relationship, since our objective here is to experimentally understand the significance of texture at high spatial resolution on AGB estimation. The AGB is measured in Tons $(\mathrm{T})$ or $(\mathrm{kg})$ over the size of the test area, which in our case is equivalent to the tree crown.

\section{Results and Discussions}

In this section, the results of the study are presented. A discussion on these results is also presented along side the results. A more detailed discussion on the afromontane camphor trees is presented, as it is the most significant and dominant vegetation on this part of Mt. Kenya.

\subsection{GLCM Results}

Results from the variance estimates of 8-tested GLCMtextures were plotted for all the training samples, over the 20windows. All the 8-texture measures showed different results for the "training samples". However, there are some common observations for all the eight training samples: (a) the texture measure with the least variance was consistently recorded as the angular second moment (ASM or S-Moment), (b) the second and third least variances are the homogeneity and entropy textures, (c) the variance texture generally exhibited the highest variance measure, followed closely in some cases by contrast, and (d) the correlation texture is parallel to the angular second moment texture, except for the soil and clouds. The rest if the textures were mixed in the order of their variance magnitudes. As hypothesized in the methodology, the ASM results were utilized to model or determine the optimal window size using variogram geostatistics. These results were then applied to the rest of the GLCM texture measures.

\subsection{Variogram Evaluation of ASM over Multisized Texture Windows}

The ASM-variance over the moving windows, from the GLCM results in sub-section 4.1, is shown in Figure 4. It is observed that apart from the clouds and soil, the rest of the "training samples" had significantly low variance and reached a saturation point after around the first nine or so windows. Clouds and bare soil are homogeneous covers whose spatial scale may be explained in terms of grain sizes and do not easily converge at some window or point. For this reason it may be difficult to directly visually determine the optimal scales for texture mapping. Such surfaces require some modeling

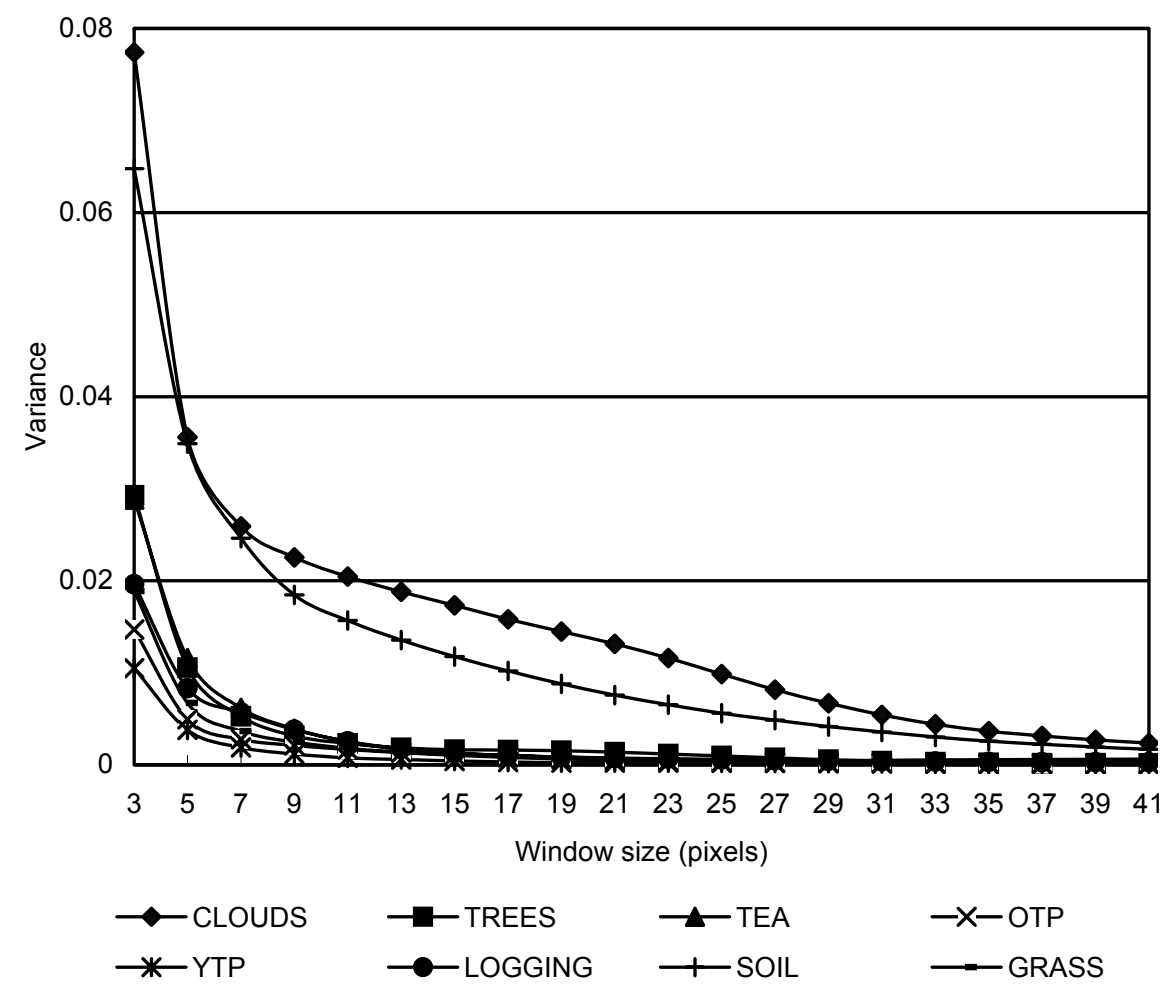

Figure 4. ASM-"training sample" variances over the texture windows. 
like semivariance fitting. Other land covers like trees have unique sizes, which can be guessed directly through some experimentation. The problem is that in trying to guess the optimal window sizes we realized that for each class, single window determination was not possible. Thus for the test afromontane trees, we illustrate its variance variability over the 20-moving windows (Figure 5a) and present the results of semivariance-modeled threshold or optimal window along side (Figure 5b).

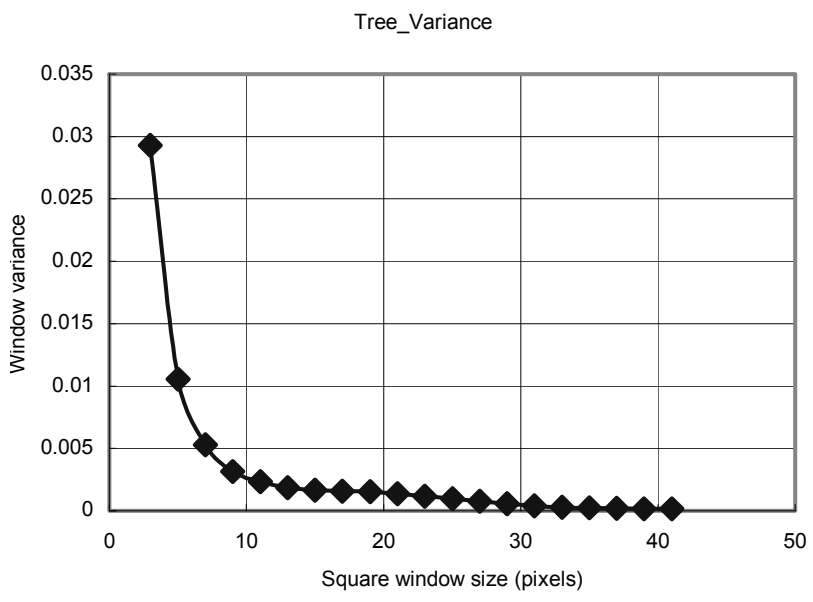

(a)

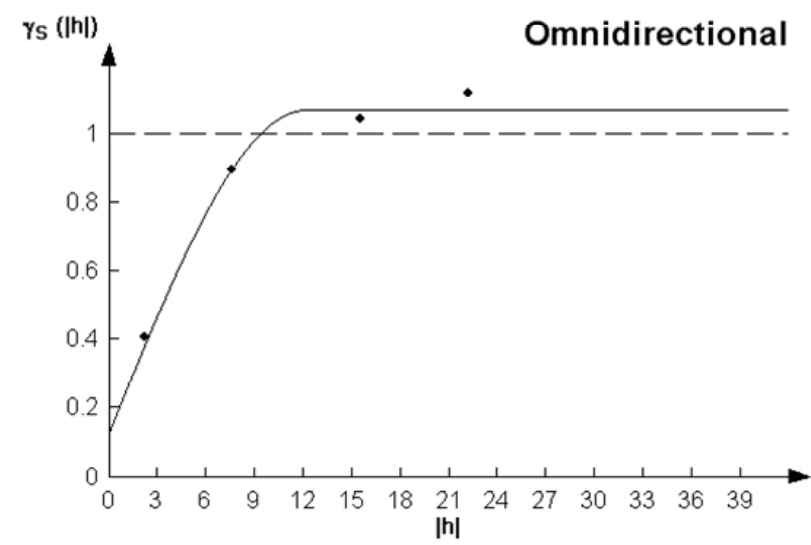

(b)

Figure 5. Variance and standard semivariance fitting of ASM texture for optimal texture window size determination for the afromontane test tree.

In each case the Spherical, the Gaussian and Exponential models were fitted to the variograms. The spherical fit gave the best model fit based on the goodness of fit results presented in Table 2 for the "training samples". The results indicate that without some kind of optimization like the semivariance window size modeling, it might not be possible to directly select on unique window. This observation can be supported by the fact that a single window cannot represent real world landscape features or components. However for actual application purposes, the objective is to determine that optimal window with the maximum information and least noise.

The results of the semivariance optimization are summarized in Table 2. The representative optimal window sizes from these results are either even or odd with decimal window sizes. One way of obtaining the optimal window size would be to take the approximate value or the nearest odd number. However, there is no physical meaning to justify this kind of "trial-and-error" window selection. We thus introduced the idea of multiple window sizes integration. Thus for an optimal window size $s_{j} \times s_{j}$ and texture model $\tau_{k}$, we intuitively take the two window sizes: $s_{j-1} \times s_{j-1}$ and $s_{j+1} \times s_{j+1}$, where the window size is even and lies approximately in the middle between the two odd sized windows. Otherwise the nearest odd window size is selected. The results of this intuitive reasoning are presented in Table 2 for each "training class".

The results in Table 2 show that clouds, trees and logged areas required two optimal windows. For these classes, even the windows were approximated to be the best, by taking the average of the minimum and maximum as illustrated in the approximation (approx.) column. Thus for the afromontane trees, the $12 \times 12$ window size was approximated as the optimal for texture mapping. Clouds and bare soil had the largest optimal window sizes as compared to afromontane trees with the least window size. Old (OTP) and young (YTP) trees, tea, soil and grass could be mapped using a single approximated window. YTP and tea exhibit closely related textural patterns and also have the same optimal window size. This may be attributed to their closeness in height, size and compactness of respective crowns. Grass also has the same optimal window size as tea and TYP, however its textural structure as seen in Figure 4 is slightly different.

If we were to empirically determine the optimal texture bands, from the variance of the ASM plots directly as shown in Figure 5a, then the multiple window concepts for some of "training samples" is a reality. The drawback to this empirical selection is that it is time-consuming and there is high likelihood of errors due to subjective estimations.

The dependence of some of the "training samples" on multiple windows may be based on the fact that each window captures a feature of a particular size, which is constituted by pixels that are independently captured by the reflectivity of one or several micro-textured surfaces within the "training sample" area. Therefore, the same texture method may produce significantly different values when it is applied to a small window or to a large one, though both windows are centered at the same pixel. This implies that the corresponding contributions of the two selected windows may also be different and uncorrelated.

It was found that selection of the optimal window size was only possible in some cases by considering both the minimum (min), average (approx) and maximum (max) windows as illustrated in Table 2. It may be concluded that the variogram determined optimal window should be used as an estimator to the maximum window size for the particular land cover considered. 
Table 2. Descriptive Statistics for "Training Samples" Standard Semivariance Fitting

\begin{tabular}{|c|c|c|c|c|c|c|c|c|}
\hline \multirow{2}{*}{$\begin{array}{l}\text { Training } \\
\text { sample }\end{array}$} & \multirow[t]{2}{*}{ Range } & \multirow[t]{2}{*}{ Sill } & \multirow[t]{2}{*}{ Nugget } & \multirow{2}{*}{$\begin{array}{l}\text { Best fit } \\
\text { model }\end{array}$} & \multirow{2}{*}{$\begin{array}{l}\text { Goodness of } \\
\text { fit found }(F)\end{array}$} & \multirow{2}{*}{$\begin{array}{l}\text { Selected } \\
\min \end{array}$} & \multirow{2}{*}{$\begin{array}{l}\text { Square } \\
\text { approx. }\end{array}$} & \multirow{2}{*}{$\frac{\text { Windows }}{\max }$} \\
\hline & & & & & & & & \\
\hline Clouds & 22.006 & 1.0000 & 0.1199 & Spherical & $2.3836 \mathrm{e}-03$ & $21 \times 21$ & $22 \times 22$ & $23 \times 23$ \\
\hline Trees & 12.123 & 0.9399 & 0.1300 & Spherical & $1.2491 \mathrm{e}-03$ & $11 \times 11$ & $12 \times 12$ & $13 \times 13$ \\
\hline OTP & 14.924 & 0.9900 & 0.1499 & Spherical & $7.2938 \mathrm{e}-03$ & & $15 \times 15$ & \\
\hline YTP & 13.074 & 0.9699 & 0.1399 & Spherical & $6.4794 \mathrm{e}-04$ & & $13 \times 13$ & \\
\hline Tea & 13.342 & 1.0000 & 0.0900 & Spherical & $9.7408 \mathrm{e}-04$ & & $13 \times 13$ & \\
\hline Soil & 19.093 & 1.0000 & 0.0050 & Spherical & $8.9166 \mathrm{e}-03$ & & $19 \times 19$ & \\
\hline Logging & 13.612 & 0.9999 & 0.0600 & Spherical & $5.4590 \mathrm{e}-03$ & $13 \times 13$ & $14 \times 14$ & $15 \times 15$ \\
\hline Grass & 12.415 & 0.9800 & 0.1000 & Spherical & $5.0525 \mathrm{e}-03$ & & $13 \times 13$ & \\
\hline
\end{tabular}

Table 3. Summary Statistics of Camphor Tree in the " $12 \times 12$ " Window Texture Measures

\begin{tabular}{llllll}
\hline Texture measure & Min & Max & Mean $(\mu)$ & Standard Deviation $(\sigma)$ & Energy $($ ENE) \\
\hline Mean & 10 & 28 & 19.747 & 4.547 & 24,360 \\
Variance & 6 & 91 & 28.240 & 16.147 & 8,920 \\
Homogeneity & 0 & 0 & 0.000 & 0.000 & 360,000 \\
Contrast & 5 & 33 & 12.645 & 5.932 & 22,590 \\
Dissimilarity & 1 & 4 & 2.063 & 0.624 & 170,194 \\
Entropy & 3 & 4 & 3.985 & 0.122 & 349,362 \\
Second Moment & 0 & 0 & 0.000 & 0.000 & 360,000 \\
Correlation & 103 & 126 & 121.157 & 3.845 & 37,284 \\
\hline
\end{tabular}

A summary of the statistics for the optimal window over the afromontane tree is presented in Table 3 for all the textures. The minimum (min), maximum ( $\max )$, mean, standard deviation and energy over the tree are computed. It is observed that over the same tree, some texture measures like homogeneity and second moment gave zero-value statistics. The variance based texture measure gave the highest variability of 85 by comparing the min and max $\mathrm{DN}$ values, and also the highest standard deviation over the tree. The correlation gave a remarkably high mean, of course based on the high min and max DN values.

Another suitable estimator of the relevant information contained within a texture image is the energy measure over an optimal window. Energy depicts the general information about the image, by indicating how much detail is present in the whole image and is a measure of the disorder within the image. We constructed a histogram of the frequencies of occurrence $\left(P_{i}\right)$ for digital numbers $(i)$ associated with individual pixels, and then calculated energy (ENE) as follows:

$E N E=\sum_{i=1}^{\max } P_{i}^{2}$

The results for the energy computations are also presen- ted in Table 3. The results show that homogeneity and second moment texture measures have the highest and equal energy. This is followed closely by entropy and then dissimilarity and the least being the variance. These results indicates that the higher the standard deviation the lower the energy and vice versa within the optimal window.

By plotting and comparing the histograms derived from the NIR bands and the 8-GLCM texture measures over the afromontane (camphor) trees, it is observed that the NIR reflectance shows a typical deciduous tree crown reflectance, whereby the center of the crown is the peak. It was seen that each texture presents a different type of information regarding pixel distributions, in some cases different from the spectral signatures, and in other cases trying to replicate the spectral patterns like the mean-texture histogram. Variance, contrast and dissimilarity texture measures exhibited nearly the same texture patterns, except for a shift in the histogram peak as compared to the NIR. Correlation and entropy texture patterns show a closely related pattern that is nearly the opposite of the variance, contrast and dissimilarity. Homogeneity and second moment present have similar results with zero values, which can physically be attributed to no information. However, it is not directly conclusive from Table 3 results, as to which texture measure is suitable for the biomass estimation. Further robust regression measures need to be carried out in order to 


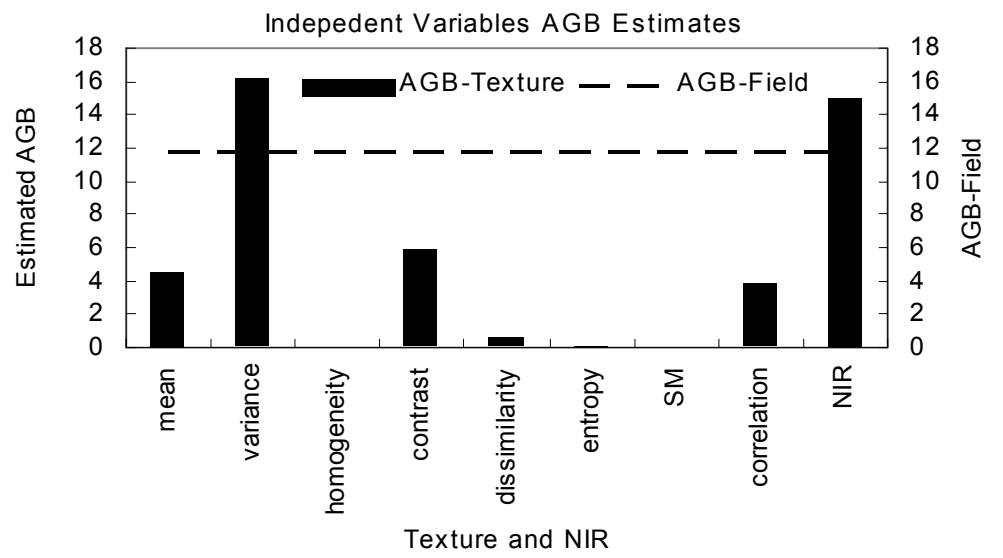

Figure 6. Comparison of texture and NIR bands against field estimated AGB results.

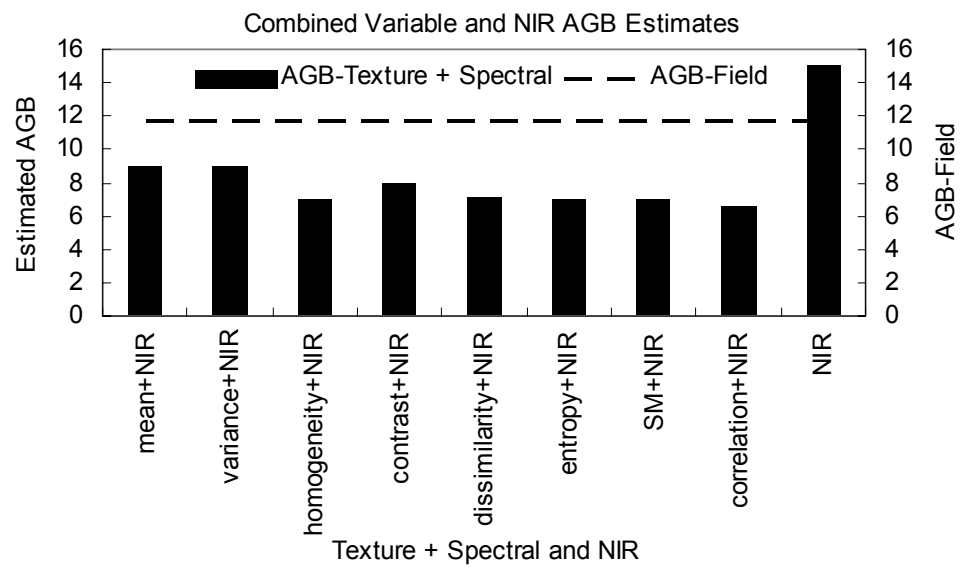

Figure 7. Comparison of texture combined with NIR, NIR band and field estimated AGB results.

determine the actual degree of the relationship between texture estimated AGB and field (actual) derived AGB.

\subsection{AGB Estimation Using Texture Measures-Afromon- tane Camphor Trees}

Using the physically derived biomass for the approximately $530 \mathrm{~m}^{2}$ crown sized tree, we determined the AGB as of $11.73 \mathrm{~T}$. The estimated AGB is compared to the 8-texture measures in order to determine which of the texture measure(s) gives the best regression with biomass. Note that in this study we are not directly concerned with the AGB estimation models, but rather with the demonstration of the significance of the texture. The differences between the field estimated AGB and the AGB estimated from NIR band and the texture measures are presented in Figure 6. As independent variables, the NIR band gave the best results, with approximately $28.0 \%$ error (overestimate) as compared to the texture measures. The results in Figure 6 indicate that among the textures, the variance texture is the best AGB estimator, even though it overestimated the AGB by relative error amount of (37.66\%). Ho- mogeneity and second moment gave the worst results with no estimate at all of the AGB. The dissimilarity and entropy also poorly underestimated the AGB, both by over $90 \%$ error. The mean and correlation showed a relative error of above $60 \%$ and the contrast had a modest relative error of about $50 \%$. The results shows that the textures by themselves may not be suitable for direct AGB estimation as they report large error magnitudes compared to the NIR and ground (actual) estimates. This may be attributed to the fact that texture measures only present the spatial information, which are not directly measured by the sensors. The spectral information is directly measured by the sensors and thus most likely to carry more obvious information that is relevant to and significant in AGB estimation or even modeling.

Relating the results in Figure 6 to those in Table 3, it is conclusive that the lower the texture energy the higher the standard deviation or variance of the texture image and the better it is for AGB estimation. This means that the higher energy depicts a large amount of disorder existing among the pixel values, and the lower standard deviation means that the pixel value frequency distribution has less dispersion. The va- 
riance, texture as an independent variable gave the best or optimal results and least was from homogeneity and second moment textures. The biomass-texture estimates were then combined with the NIR to dependably assess the contribution of spatial information in biomass estimation upon combination with spectral information in high-resolution imagery.

\subsection{Integration of Texture with NIR for AGB Estimation - Afromontane Camphor Trees}

Though it is possible to conclude from the results in section 4.3 that the variance gave the best results, we continued to compare all the textures upon integration with the NIR band. The results of the comparisons between NIR alone and its combination with the textures are presented in Figure 7. Comparing the results in Figures 6 and 7, the immediate and obvious conclusion is that spectral information plays a more significant role in AGB estimation than texture, if compared as independent variables. It is not because NIR has a higher regression with actual field measured AGB estimates, but because its combination with textures significantly improved AGB estimation for all the textures as compared to when they were assessed independently. However, we must note that this observation does not rule out the possible contribution of texture in improving the accuracy with which the AGB is measured in another case study.

The results show that upon combination with the spectral (NIR) information, the variance and mean now perform better than NIR band alone, by improving the accuracy of biomass estimation by approximately $4.82 \%$ for the mean and $4.33 \%$ for the variance. While the AGB estimation is remarkable improved on the combination of mean texture with the NIR band, it was marginally so for the variance texture. It is conclusive from these observations that not all textures contribute positively towards improving the accuracy with which AGB is estimated from remote sensing data.

Comparing the regressions between the field AGB, the independent variables (bands) and combined variables listed in Table 4, it is seen that the NIR band was instrumental in improving the AGB estimation for all the textures and most significantly so for; homogeneity, dissimilarity, entropy and second moment, from nearly zero to about $40 \%$. This made the correlation, which was initially as good as the mean to become the least estimator among the textures. The results (Table 4) show that for this kind of afromontane camphor tree, the homogeneity, entropy and second moment arguably gave similar results. The same can be applied for the mean and variance textures. Only the mean and variance had higher regressions $\left(R^{2}>0.76\right)$ than NIR band $\left(R^{2}=0.72\right)$. As dependent variables, all the textures underestimated the AGB on combination with NIR. However independently the variance and the NIR overestimated AGB.

From these results, it can be said that there is no direct dependency or relationship between the independent texture variables and their improvements for the AGB estimation, on combination with the NIR band. Instead for each scene, this kind of relationship must be derived independently. The inter- action between texture and spectral information may not also be easily or directly predictable, except to state the amount of improvement or decrement for AGB estimation. For example while some textures with very low regressions $\left(R^{2}<0.50\right)$ cannot be independently used to estimate AGB, their combination with NIR improves AGB estimation by more than $50 \%$ as the case for homogeneity, entropy and second moment, but lowers the performance of the NIR. The results in Table 4 also suggest that the independent variables (spectral and spatial) do not have sufficiently high regression coefficients to develop biomass estimation models.

Table 4. Regression between the Field AGB, Independent Variables and Combined Variables

\begin{tabular}{lll}
\hline \multirow{2}{*}{ Variable } & \multicolumn{2}{c}{ Regression $\left(\mathrm{R}^{2}\right)$} \\
\cline { 2 - 3 } & Single variable & Texture + NIR \\
\hline Mean & 0.388 & 0.768 \\
Variance & 0.623 & 0.763 \\
Homogeneity & 0.000 & 0.597 \\
Contrast & 0.506 & 0.676 \\
Dissimilarity & 0.053 & 0.607 \\
Entropy & 0.011 & 0.597 \\
Second Moment & 0.000 & 0.597 \\
Correlation & 0.328 & 0.560 \\
NIR & 0.720 & N/A \\
\hline
\end{tabular}

It can be conclusive that the roles of suitable texture measures in AGB estimation is valuable and significant since appropriate texture may offer and effective method for improving model performance. Therefore it is necessary to seek two or more independent variables, such as in this study, to combine texture and NIR in order to improve the relationships between biomass and remotely sensed data. Figure 8 summarizes the best results that were obtained with mean and variance in this study. The slight difference between the mean and variance can be related to fact that the mean-NIR combination closely replicated the spectral and spatial structure of the tree than the variance-NIR combination (compare Figure 8 and Table 4).

In a summary from the above results, the difference between the textures upon integration with spectral information from the NIR has a lower disparity compared to when the textures are analyzed independently. It is worth noting that a care must be taken while using the GLCM for biomass estimation. This is because random selection of texture may lead to poor results as demonstrated by the results of this study. It may be said that a texture method that greatly "deforms" the original spectral pattern may not be suitable in this particular case of afromontane trees, which could be reason why the mean-texture gave overall best results on combination with NIR band.

\subsection{AGB Estimation-Tea, Young and Old Planted Trees}

The results for comparison between the AGB estimates from NIR, the texture bands and a combination of the two ag- 

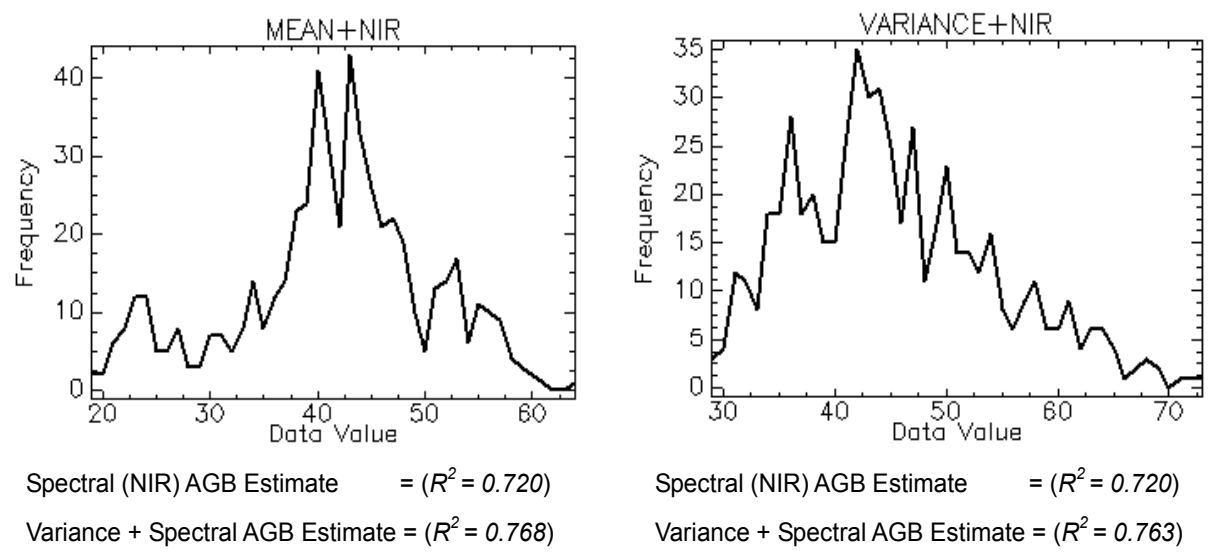

Figure 8. Summary statistics of the mean and variance results for AGB estimation.

ainst the field measured AGB for tea, young and old pine trees are presented in Figure 9. The field estimated AGB biomass for these land cover over the test area are $6.502 \mathrm{~kg}$ for tea, $7.505 \mathrm{~kg}$ for young pine trees (3.5 years old) and $9.779 \mathrm{~kg}$ for the older pine trees ( 6 years old). These AGB estimates are far less than those of the natural fully grown camphor trees as may be expected.

For the case of tea, Figure 9a, the NIR gave the best results are independent spectral variable while with texture, variance correlation and contrast gave very close and comparably the best results. All the textures grossly underestimated AGB as compared to the field-estimates. The lowest close zero estimates were obtained from the homogeneity, entropy and second moment. Upon combination with spectral (NIR) information, all the textures overestimated the AGB by slightly more than $30 \%$ in each case. The best estimates whose errors were also comparable to that of NIR alone were the least as independent variables (homogeneity, entropy and second moment). In overall for tea, the GLCM texture information did not seem to be quite significant in biomass estimation. Instead all of the textures upon combination with NIR gave nearly the same results are NIR in itself. This may be attributed to the fact that probably the textures tested are not suitable for this kind of vegetation or to be more specific planted and well structured vegetation like tree.

Looking at the older planted pine trees results in Figure $9 \mathrm{~b}$, the independent variables contrast outperformed all the textures and also the spectral (NIR) band. NIR was the second best and overestimated AGB as compared to all the textures that underestimated the AGB except the variance texture. The underestimation errors in the cases of homogeneity, dissimilarity, entropy and second moment were more than $95 \%$, implying that they were unsuitable for the estimation of AGB for this particular vegetation type. On the contrary, when the spectral and spatial information are combined, all the combinations gave nearly a same error range of approximately $32 \%$, except for the variance $(67.25 \%)$, the contrast $(56.79 \%)$ and the mean $(42.19 \%)$. The results from NIR as an independent variable and when combined with homogeneity and second moment gave the same and best results with error of $32.11 \%$. This implies that whether these two textures are included or not the optimal results would still be obtained from the spectral information alone. Contrast texture is seen in this case to give the best results $\left(R^{2}=0.753\right)$ in this case as independent variable, yet upon combination with spectral the regression drops to $\left(R^{2}=0.432\right)$.

In Figure 9c, the results for the young planted pine trees are presented. As the case for the older pine trees, it is observed that the correlation, variance and contrast, in that order, gave the best results are independent variables. Spectral (NIR) information was fourth though with very large error of more than $75 \%$, like the reminder of the textures. Upon combination between the textures and NIR band, the results are dramatically worsened. All the bands present very poor results $\left(R^{2}<\right.$ $0.25)$, with similar results to NIR band alone from homogeneity, dissimilarity, entropy and second moment. The worst results among the combination came from variance. In case of younger pine trees, correlation, variance and contrast texture seemed a play a much more significant role in AGB estimation as independent variables than spectral information, either as independent or combined variable.

This research indicates that texture information is a very important factor in improving biomass estimation and might also be significant in estimation model performance especially in tropical rainforests. It must however be pointed out that it is a difficult task to find the appropriate texture measure(s) that that are strongly related to biomass because only some texture measures with a specific window size and from specific image spectral channels can effectively extract biomass information. Another point is that for some vegetation types, texture may not always perform well independently and thus its combination with the appropriate band(s) may greatly improve its significance or better the accuracy of the results. On other cases, the texture alone or spectral information alone are sufficient. However this must be tested and we anticipate that results can differ in different study areas. Further research on integrating 

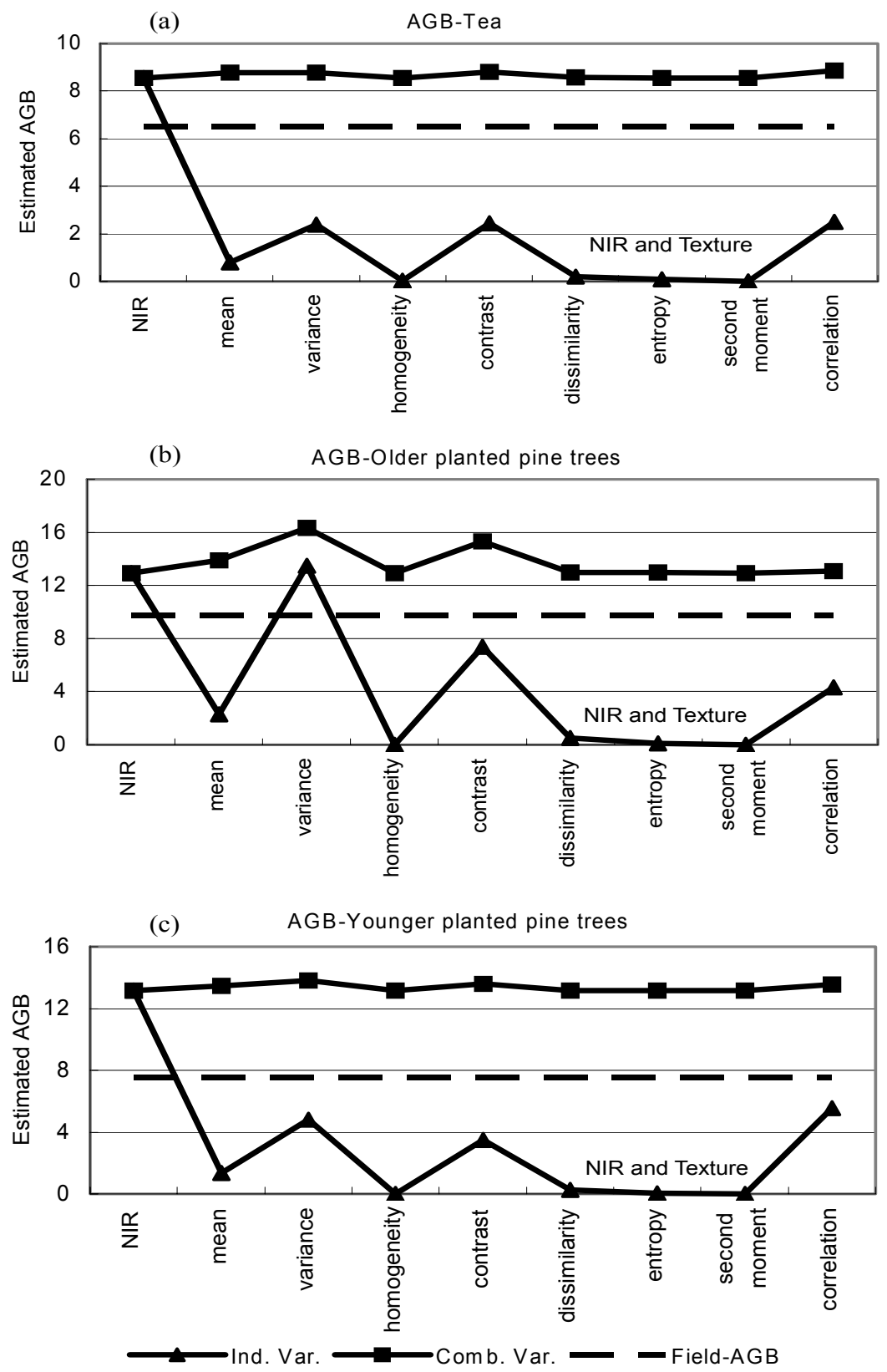

Figure 9. AGB estimate comparisons for tea, old and young pine trees.

and comparing the results of this study with vegetation indices is necessary in future.

From above results, it was observed that for AGB estimation in high spatial resolution data, texture plays a crucial role that can best be understood through comparing different: texture types, optimal window sizes, vegetation species and combination with spectral information. Different conclusions on the role of texture are inferred for different tree or vegetation types meaning that generalization may not be application. Compared to the natural camphor trees, these three vegetation types are far younger in age and size. Since they are planted in patterned rows, they exhibit a specific texture structure compared to the natural trees. This type of texture structuring may not be captured accurately or fully by the GLCM methods, in the case of planned trees. For this reason, the GLCM textures performed much more poorly in estimated AGB for these three vegetation types than for the natural camphor trees. We may conclude here that the performance of GLCM texture depends on the tree species and the inherent structural arrangement of that particular species. We suggest here that a more 
direction based texture method may be more appropriate for planned forested environments than for natural forest environments.

\section{Conclusions}

Image spatial information, texture, has been suggested as one of the clues towards improving the extraction of biophysical parameters like biomass from high-resolution imagery. A wide variety of texture feature extraction methods have been proposed for texture based image classification and segmentation. These methods are typically evaluated over windows of the same size, the latter being usually chosen for each particular method on an experimental basis. However for biomass extraction, little has been achieved. The proposed texture optimization approach in this study can be useful for estimating the above ground biomass on integration with spectral information for specific scene and specific vegetation type. The approach is useful for selecting appropriate textural signature(s) and window(s) for combination with spectral information, and offers the possibility for suitable AGB estimation model formulation. The effectiveness of texture measure can be argued to be strongly dependent on the vegetation species and the corresponding biophysical characteristics. The particular finding that an estimation comprising of spectral and textural signatures provides better accuracy deepening on the selected feature, is valuable in the local biosphere-atmosphere experimentations within forest ecosystems. In the moist tropical forests like Mt. Kenya, there is difficulty in gathering sufficient ground-truth data representative over a large area. Thus such optimization models are cost-effective and suitable for simulating and estimating the biomass distribution information and biomass statistical data in a timely and accurate manner.

We concluded that the role of texture type and optimal window in AGB estimation depends on the: size (height), age, species, inherent spatial structure (natural or planted) and crown size of the vegetation species. Further work will consist of the combination or integration different texture methods for the same task of AGB estimation, in a much wider geographic area. Finally, to improve on the regression between the field and estimates from remote sensing data, we suggest that alternative sensors based on LiDAR and InSAR ought be compared and integrated with optical sensors, as they provide details on the vertical (3D) spectral and spatial details of vegetation.

Acknowledgments. The authors wish to gratefully acknowledge the grants from the Japanese Government-Ministry of Education and Science for this research.

\section{References}

Bailey, G.B., Lauer, D.T. and Carneggie, D.M. (2001). International collaboration: The cornerstone of satellite land remote sensing in the 21st century. Space Policy, 17, 161-169.

Brown, S.L., Schroeder, P. and Kern, J.S. (1999). Spatial distribution of biomass in forests of the eastern USA. For. Ecol. Manage.,
$123,81-90$.

Chang, K.I., Bowyer, K.W. and Sivagurunath, M. (1999). Evaluation of texture segmentation algorithms, in Proc. of IEEE Computer Vision and Pattern Recognition, Fort Collins, USA, pp. 94-299.

Chen, J.M., Pavlic, G., Brown, L., Cihlar, J., Leblanc, S.G., White, P., Hall, R.J., Peddle, D., King, D.J., Trofymow, J.A., Swift, E., Van Der Sanden, J. and Pellikka, P. (2002). Validation of Canada-wide LAI maps using ground measurements and high and moderate resolution satellite imagery. Remote Sens. Environ., 80, 165-184.

Crow, T.R. and Schlaegel, B.E. (1988). A guide to using regression equations for estimating tree biomass. Northern J. Appl. For., 5, $15-22$.

Curran, P. and Atkinson, P. (1998). Geostatistics and remote sensing. Prog. Phys. Geogr., 22, 61-78.

Foody, G.M., Boyd, D.S. and Culter, M.E.J. (2003). Predictive relations of tropical forest biomass from Landsat TM data and their transferability between regions. Remote Sens. Environ., 85, 463474.

Franklin, J. and Hiernaux, P. (1991). Estimating foliage and woody biomass in Sahelian and Sudanian woodlands using a remote sensing model. Int. J. Remote Sens., 12, 1387-1404.

García-Sevilla, P. and Petrou, M. (2000). Analysis of irregularly shaped texture regions: A comparative study, 15th IAPR International Conference on Pattern Recognition, Barcelona, pp. 1080-1083.

Haralick, R.M., Shanmugam, K. and Distein, I. (1973). Textural features for image classification. IEEE Trans. SMC, 6(3), 610-622.

Jakubauskas, M.E. (1996). Thematic Mapper characterization of lodgepole pine serals in Yellowstone National Park, USA. Remote Sens. Environ., 56, 118-132.

Lee, N.J. and Nakane, K. (1997). Forest vegetation classification and biomass estimation based on Landsat TM data in mountainous region of west Japan, in H.L. Gholz, K. Nakane and H. Shimoda (Eds.), The Use of Remote Sensing in the Modeling of Forest Productivity, Dordrecht7 Kluwer, pp. 159-171.

Nelson, R.F., Kimes, D.S., Salas, W.A. and Routhier, M. (2000). Secondary forest age and tropical forest biomass estimation using Thematic Mapper imagery. J. Biosci., 50, 419-431.

Olthof, I. and King, D.J. (2000). Development of a forest health index using multispectral airborne digital camera imagery. Can. J. Remote Sens., 26, 166-175.

Pannatier, Y. (1993-1996). Variowin: Software for Spatial Data Analysis in $2 D$ (Software), Springer-Verlag, New York, USA.

Paruelo, J.M. and Lauenroth, W.K. (1998). Interannual variability of NDVI and its relationship to climate for North American shrublands and grasslands. J. Biogeogr., 25, 721-733.

Randen, T. and Husoy, J.H. (1999). Filtering for texture classification: A comparative study. IEEE Trans. PAMI, 21(4), 291-310.

Read, J.M., Clark, D.B., Venticinque, E.M. and Moreira, M.P. (2003). Application of merged $1-\mathrm{m}$ and 4-m resolution satellite data to research and management in tropical forests. J. Appl. Ecol., 40(3), 592-600.

Ryu, S.R., Chen, J., Crow, T.R. and Saunders, S.C. (2004). Available fuel dynamics in nine contrasting forest ecosystems in North America. Environ. Manage., 33, 87-107.

Sannier, C.A.D., Taylor, J.C. and Plessis, W.D. (2002). Real-time monitoring of vegetation biomass with NOAA-AVHRR in Etosha National Park, Namibia, for a fire risk assessment. Int. J. Remote Sens., 23, 71-89.

Soares, J.V., Rennó, C.D., Formaggio, A.R., Yanasse, C.C.F. and Frery, A.C. (1997). An investigation of the selection of texture features for crop discrimination using SAR imagery. Remote Sens. Environ., 59, 234-247.

Steininger, M.K. (2000). Satellite estimation of tropical secondary forest above-ground biomass: Data from Brazil and Bolivia. Int. J. Remote Sens., 21, 1139-1157. 